\title{
Influence of Maglev Force Relaxation on Hysteresis of Bulk HTSC Above the NdFeB Guideway at Different Lateral Displacements
}

\author{
Yujie Qin · Yunxing Shu
}

Received: 26 April 2010 / Accepted: 2 June 2010 / Published online: 3 July 2010

(C) The Author(s) 2010. This article is published with open access at Springerlink.com

\begin{abstract}
After the maglev force (both levitation and guidance forces) relaxation property of a high-temperature superconductor (HTS) above a permanent-magnet guideway (PMG) was studied experimentally, the HTS bulk was horizontally moved in the lateral direction with different displacements above the permanent-magnet guideway. Both levitation and guidance force curves with lateral displacement were obtained by a SCML-2 measurement system synchronously. It was found that the levitation force hysteresis after relaxation was reduced compared to the case without relaxation, while the guidance force hysteresis curve almost did not change. This study provided a scientific analysis for the practical application of the bulk HTS.
\end{abstract}

Keywords Type-II superconductor · YBCO - Time relaxation $\cdot \mathrm{NdFeB}$ guideway $\cdot$ Hysteresis

\section{Introduction}

After the discovery of stable levitation of a bulk high-Tc Superconductor (HTS) above (or below) a permanent magnet, high-temperature superconductor (HTSC) bulk samples have been widely used on many applications, such as in noncontact bearings, flywheel energy storage systems, magnetic levitating (maglev) transportation systems, rotary and linear motors and generators [1-6]. In particular, our previous reports have shown great advances in the area of maglev technology using bulk HTSC samples for the guiding and sus-

\section{Y. Qin $(\bowtie) \cdot$ Y. Shu}

Department of Computer and Information Engineering, Luoyang Institute of Science and Technology, Luoyang, Henan 471023, People's Republic of China

e-mail: qyjswjtu@vip.sohu.com pending of the maglev vehicle above a permanent-magnet guideway (PMG) [7].

Two key parameters stand out the most when studying the mentioned maglev system, the vertical levitation force (FZ) and the lateral guidance force (FX) experienced by the vehicle, which are usually either studied experimentally, theoretically and/or computationally. Theoretically speaking, there are two parameter components that make up the integral Lorentz force which results from the interaction between the induced currents and the applied external magnetic field. This paper studied the magnetic force relaxation combining the two parameters from the point of view of practical applications. Henceforth, the influence of the relaxation of both FZ and FX on the hysteresis losses of the bulk HTSC above the NdFeB PMG with different lateral displacements was investigated. During practical applications, the vehicle's body may become off-centered from the PMG's centerline for reasons such as field non-uniformity due to cooling inhomogeneity or external disturbances. Therefore, it is vitally important to study the maglev characteristics of the bulk HTSC with different lateral movements for the successful engineering application of the HTS maglev vehicle [8-10].

\section{Experimental}

The bulk HTSC used was a single-domain YBCO sample shaped like a disc having dimensions of $50 \mathrm{~mm}$ in diameter and $15 \mathrm{~mm}$ in thickness with the c-axis aligned in the thickness direction. The SCML-02 measurement system, described elsewhere [11], was employed to measure FZ and FX exerted by the HTSC bulk.

The structure and the magnetic field distribution at a height of $g=3 \mathrm{~mm}$ of the PMG is shown in Fig. 1, similar to the guideway used in [12]. 


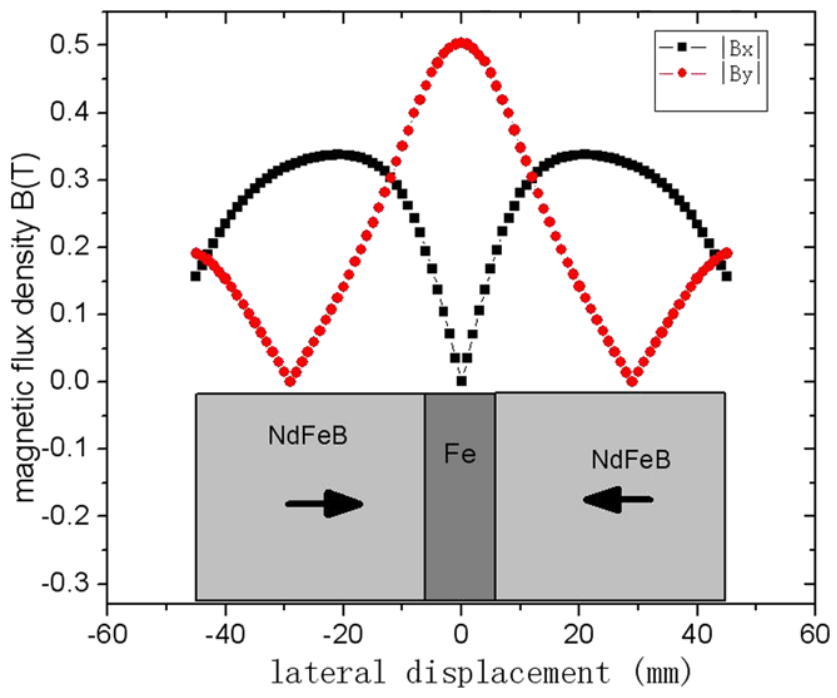

Fig. 1 PMG structure and magnetic field distribution at a height of $3 \mathrm{~mm}$ above the PMG

The experimental processes taken to investigate the relaxation and hysteresis behavior of FZ and FX are as follows.

The sample had been first immersed in liquid nitrogen $30 \mathrm{~mm}$ above the PMG (distance between the bottom of the sample and the surface of the PMG) for ten minutes to make sure the sample has transitioned to the superconducting state under field-cooling conditions. The sample was then lowered to a working height of $W H=20 \mathrm{~mm}$ and subsequently driven by a motor along the lateral direction ( $x$-axis) to displace the sample off the centerline of the PMG by $\delta=3 \mathrm{~mm}$. Immediately afterwards, FZ and FX were measured simultaneously for $600 \mathrm{~s}$, so both of the relaxation curves of the levitation force and guide force were obtained at the same time. The same process was followed to obtain each of the relaxation and hysteresis curves for $\mathrm{FZ}$ and $\mathrm{FX}$ when $\delta=5 \mathrm{~mm}$, $7 \mathrm{~mm}$ and $15 \mathrm{~mm}$ with relaxation times of $600 \mathrm{~s}$ and no relaxation.

\section{Results and Discussion}

From Fig. 2 we can see that the maglev forces (both levitation and guidance forces) relaxation property of HTS above the permanent-magnet guideway (PMG) is different. Both the levitation force and guide force of the bulk decay with time exponentially. The normalized FZ and FX curves with respect to their initial values (when $t=0 \mathrm{~s}$ ) when $\delta=3 \mathrm{~mm}$ are shown in Fig. 2. FZ decayed more rapidly than FX during the first 100 seconds for which, after the 100 second mark, FZ became more or less stable retaining $95 \%$ of its initial value, FZ(0). FX in contrast, after 100 seconds, remained decreasing, but the rate of the decrease also slowed down. $\operatorname{Fx}(600)$ was observed to have decayed by $4 \%$ of its maximum initial value.

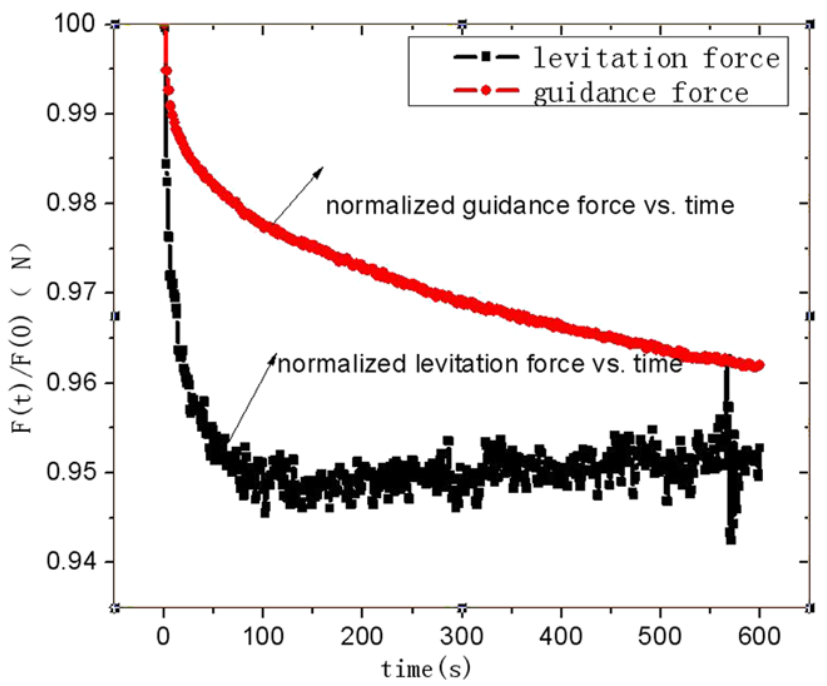

Fig. 2 Magnetic levitation and guidance forces relaxation of the HTS bulk above the PMG

Figures 3(a) and 3(b) show FZ and FX's hysteresis curves with different $\delta$ during the displacement of the HTS and right before the force relaxation. Figures $4(a)$ and (b) show the variation of levitation force and guidance force at these points during lateral displacement when $\delta=7 \mathrm{~mm}$. By the variation, we can compare the variation of levitation and guidance force's hysteresis between the cases without and with relaxation at a certain $\delta$, where $\delta=7 \mathrm{~mm}$.

From Fig. 3, we can see that the levitation force hysteresis decreases with the increasing lateral displacement. But the guidance force hysteresis increases with lateral displacement. From Fig. 4, it was found that the levitation force hysteresis after relaxation was decreased compared to without relaxation, while the guidance force hysteresis curve almost did not change. For the HTSC/PMG system in the quasistatic state, the main form of energy loss is attributed to hysteresis losses come from the hysteretic behavior of hard type-II superconductors when exposed to an applied magnetic field. And for the HTSC/PMG system, the hysteresis of the magnetic force is caused by the change of the induced currents in the HTSC bulk. Based on Maxwell's equation the induced current is related to the magnetic field difference between the applied field and the trapped magnetic field in the HTSC bulk [13]. From this point of view, we might think that the essential factor causing the energy loss here is the change of the trapped magnetic field during lateral displacement [10]. For the levitation force hysteresis corresponds to a kind of energy loss. In fact, the magnetic field in the superconductor was bound up at the centers of pinning, with the form of the magnetic flux quantum, the field into or out of the superconductor will overcome the pinning carrier energy and consume energy. This is called the levitation force hysteresis behavior. The levitation force hysteresis is related to the penetration depth. The greater the penetration depth 


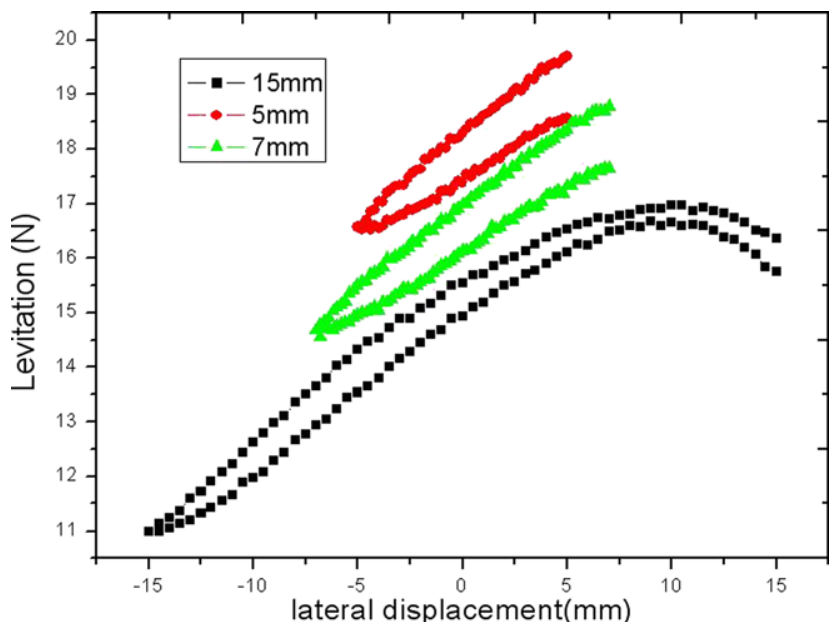

(a)

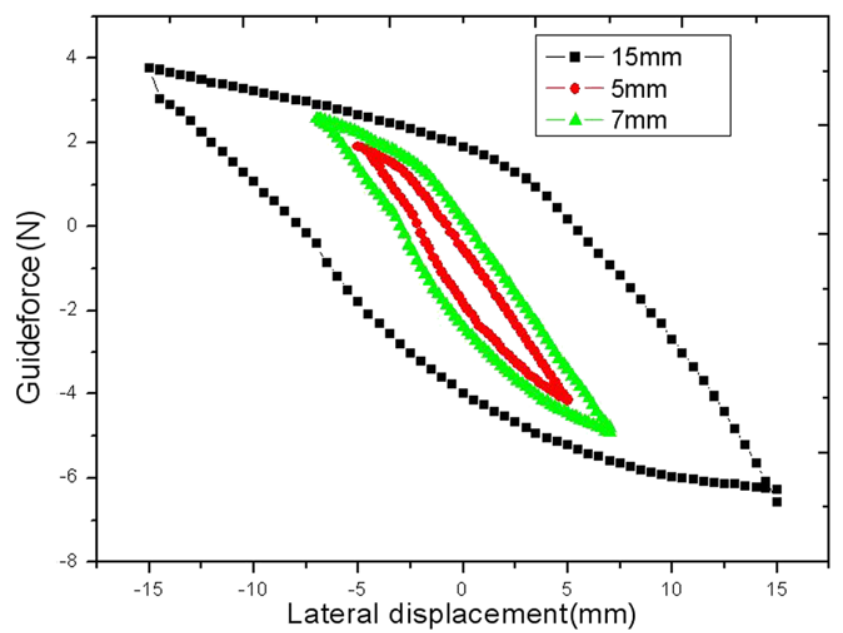

(b)

Fig. 3 Magnetic forces at different lateral displacements without force relaxation, (a) FZ, (b) FX

of the magnetic field, the more evident the levitation force hysteresis. From Fig. 2 we can see that the levitation force decay with time is shrewd at the beginning of the time, corresponding to more energy overcoming the pinning carrier. This is why the levitation force hysteresis was reduced compared to the case without relaxation. On the other hand, from Fig. 1 we can see that the applied field is alternating along the lateral direction for the horizontal component which is the field component of the generating levitation force. When the HTS bulk was horizontally moved in the lateral direction with different displacements above the PMG, the HTS bulk was remagnetized. With the lateral displacement increasing, the variation of the horizontal component of the applied field decreased, which led to a decrease of vortices in the HTS bulk, hence energy consumption. Consequently, levitation force hysteresis will decrease with the increase of the lateral displacements. As guidance force hysteresis is mainly dependent on the measurement height, the guidance force hysteresis curve almost does not change.

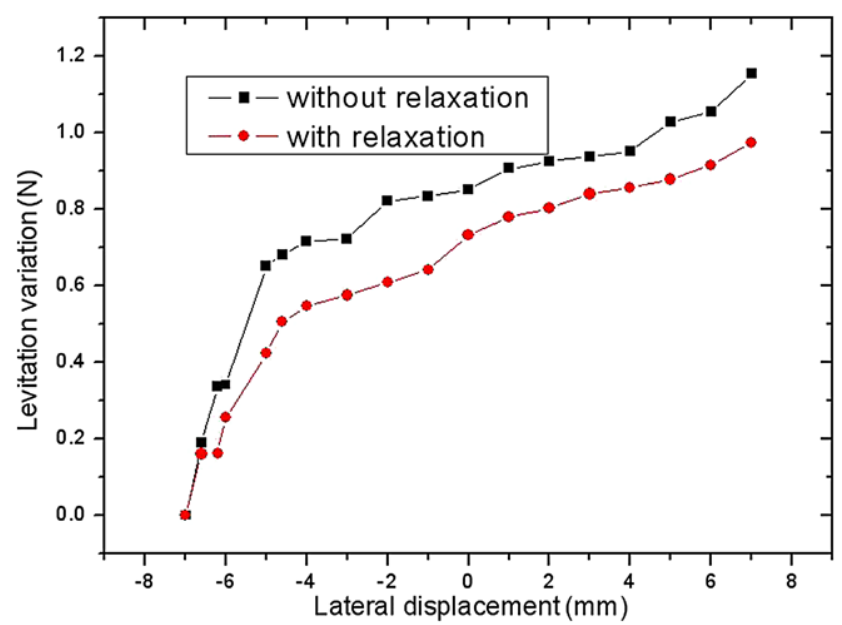

(a)

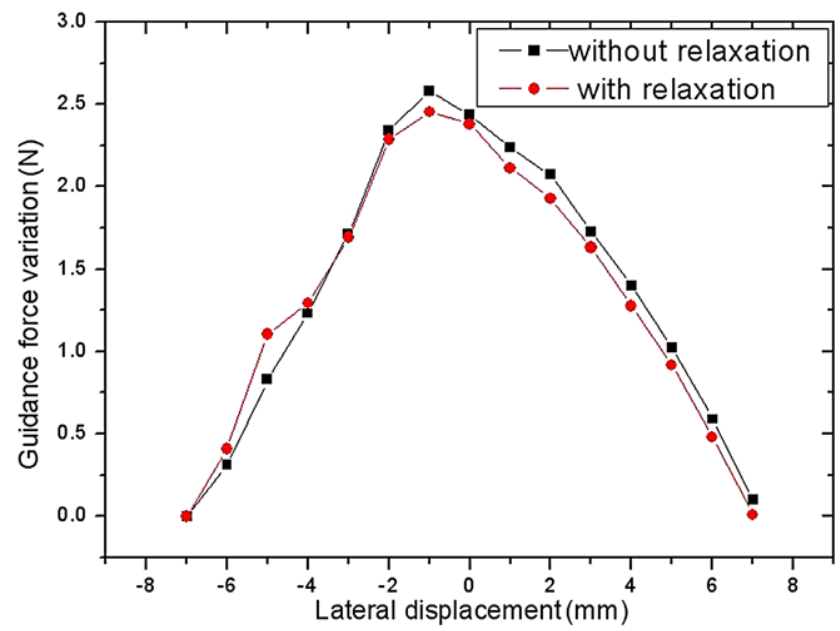

(b)

Fig. 4 Magnetic forces at different lateral displacement with force relaxation; (a) levitation force, (b) guide force

\section{Conclusions}

From the above, we can conclude to the following.

(1) The maglev forces (both levitation and guidance forces) relaxation properties of HTS above the permanentmagnet guideway (PMG) are different, while both the levitation force and guide force of the bulk decay with time exponentially.

(2) The levitation force hysteresis decreases with increasing of different lateral displacements in spite of relaxation.

(3) The influence of relaxation on both the levitation force and guide force hysteresis is different. The levitation force hysteresis after relaxation is reduced compared to without relaxation, while the guidance force hysteresis curve does not change.

Acknowledgements This work is supported by the National High Technology Research and Development Program of China 
(2007AA03Z210), the National Natural Science Foundation in China (50677057).

Open Access This article is distributed under the terms of the Creative Commons Attribution Noncommercial License which permits any noncommercial use, distribution, and reproduction in any medium, provided the original author(s) and source are credited.

\section{References}

1. Hull, J.R.: Rep. Prog. Phys. 66, 1865-1886 (2003)

2. Hull, J.R.: Supercond. Sci. Technol. 13, R1-15 (2000)

3. Strasik, M.: IEEE Trans. Appl. Supercond. 17(2), 2133-2137 (2007)

4. Izumi, M., et al.: J. Phys. Conf. Ser. 97, 012299 (2008)
5. Yang, W.J., Qiu, M., Liu, Y., Wen, Z., Duan, Y., Chen, X.D.: Supercond. Sci. Technol. 20, 281-286 (2007)

6. Murakami, M.: Physica C 341-348, 2281-2284 (2000)

7. Wang, J.S., et al.: Physica C 378-381, 809-814 (2002)

8. Del-Valle, N., Sanchez, A., Navau, C., Chen, D.-X.: Appl. Phys. Lett. 92, 042505 (2008)

9. Song, H., de Hass, O., Beyer, C., Krabbes, G., Verges, P., Schultz, L.: Appl. Phys. Lett. 86, 192506 (2005)

10. Ma, G.T., Lin, Q.X., Wang, J.S., Wang, S.Y., et al.: Supercond. Sci. Technol. 21, 065020 (2008)

11. Wang, S.Y., Wang, J.S., Deng, C.Y., et al.: IEEE Trans. Appl. Supercond. 17(2), 2091-2094 (2007)

12. Jing, H., Wang, J., Wang, S., Wang, L., Liu, L., Zheng, J., Deng, Z., Ma, G., Zhang, Y., Li, J.: Physica C 463-465, 426-430 (2007)

13. Del-Valle, N., Sanchez, A., Pardo, E., Navau, C., Chen, D.-X.: Appl. Phys. Lett. 91, 112507 (2007) 